\title{
AIDS-Related Burkitt Lymphoma
}

National Cancer Institute

\section{Source}

National Cancer Institute. AIDS-Related Burkitt Lymphoma. NCI Thesaurus. Code C8286.

A Burkitt lymphoma occurring in HIV-positive patients. 